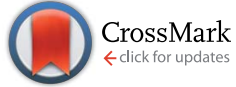

Cite this: J. Mater. Chem. A, 2015, 3, 24422

Received 15th September 2015 Accepted 6th November 2015

DOI: $10.1039 / \mathrm{cta}$ ta07408h

www.rsc.org/MaterialsA

\section{Conversion of amorphous polymer networks to covalent organic frameworks under ionothermal conditions: a facile synthesis route for covalent triazine frameworks $\uparrow$}

\begin{abstract}
Sophie Kuecken, ${ }^{a}$ Johannes Schmidt, ${ }^{a}$ Linjie Zhi $^{\mathrm{b}}$ and Arne Thomas*a
The conversion of an amorphous, non-porous polymer network into an ordered, microporous covalent organic framework under ionothermal conditions is presented. The amorphous polymer network is prepared by trimerization of carbonitrile monomers using strong Broensted acids. In a second step the polymer network is converted into a porous covalent triazine framework (CTF) under ionothermal conditions, i.e. in molten zinc chloride. This two-step process largely facilitates the synthesis of CTFs as the application of a polymeric precursor avoids early evaporation of small organic compounds at the high temperatures of the ionothermal process and therefore no evacuated and sealed quartz ampoules have to be used, which makes the CTF synthesis easier, safer and scalable.
\end{abstract}

\section{Introduction}

Microporous Polymer Networks (MPNs) and Covalent Organic Frameworks (COFs) are covalently connected, entirely organic materials which often exhibit very high permanent surface areas. ${ }^{1}$ Such materials have recently found increasing interest as their organic nature allows the incorporation of various functionalities while the covalent connections yield high thermal and chemical stability of the materials. While MPNs are amorphous, in the synthesis of COFs reversible condensation reactions are utilized enabling the formation of the thermodynamically favoured, crystalline materials. ${ }^{2}$ Covalent triazine frameworks (CTFs) are one certain type of COFs, which are formed by trimerization reaction of cyano-functionalized aromatic compounds to yield triazine linkages. The first reported CTF, CTF-1, was for example made by trimerization of 1,4-dicyanobenzene. ${ }^{3}$ Triazines are quite stable chemical compounds and to enable a reversible trimerization reaction, harsh chemical conditions, namely condensation in molten $\mathrm{ZnCl}_{2}$ at $400{ }^{\circ} \mathrm{C}$, had to be applied to receive an ordered material. So far three crystalline CTF materials have been reported (CTF-0, CTF-1 and CTF-2) ${ }^{4,5}$ plus a fully fluorinated CTF- $1 .{ }^{6}$ Also a crystalline triazine-based graphitic carbon nitride (TGCN) material has been recently prepared in salt melts. ${ }^{7}$ Numerous

${ }^{a}$ Technische Universität Berlin, Department of Chemistry, Functional Materials, Hardenbergstr. 40, 10623 Berlin, Germany. E-mail: arne.thomas@tu-berlin.de

${ }^{b}$ National Center for Nanoscience and Technology of China, No. 11, Beiyitiao Zhongguancun, 100190 Beijing, P. R. China

$\dagger$ Electronic supplementary information (ESI) available: Detailed experimental section, IR spectroscopy, thermogravimetric analysis, X-ray diffraction, nitrogen sorption of CTF materials. See DOI: 10.1039/c5ta07408h other carbonitrile compounds have been used as precursors, which however yielded amorphous triazine networks. ${ }^{8}$ CTFs have some remarkable properties which make them interesting for a number of applications. First of all, due to the stable covalent bonds and the high amount of nitrogen atoms they are extremely stable chemically as well as thermally. From TGA measurements it has been shown that CTF-0, prepared from 1,3,5-tricyanobenzene does not show any weight loss in air or oxygen until $500{ }^{\circ} \mathrm{C} .{ }^{5}$ Another CTF was applied as catalyst support for selective methane oxidation in concentrated sulfuric acid at $200{ }^{\circ} \mathrm{C}$ for several hours. The material could be recycled several times, showing no signs of decomposition. ${ }^{9}$ The abundant nitrogen atoms provide coordination sites, making CTFs attractive catalyst supports for metals. ${ }^{9-20}$ CTFs have also been applied as solid base catalysts for the conversion of $\mathrm{CO}_{2}{ }^{21}$ and as metal free catalysts for ORR. ${ }^{22,23}$ The high surface area and nitrogen amount make CTFs attractive for the storage of gases $^{6,24-28}$ or generally as sorbent materials. ${ }^{29-31}$ Furthermore, due to the conjugated planar structure, CTFs are organic semiconductors. For an ideal CTF-1 structure a band gap of $2 \mathrm{eV}$ has been calculated ${ }^{32}$ and their potential as photocatalyst has been proven theoretically ${ }^{33}$ and experimentally. ${ }^{34}$ Finally, CTFs have been recently used for sensing applications ${ }^{35}$ and as electrode materials in energy devices. ${ }^{36-40}$ It should be noted that especially for the latter applications where conductivity of materials is an important issue, most often amorphous "TTFs" (thermolyzed triazine frameworks) have been applied, for which dicyanocompounds are heated in $\mathrm{ZnCl}_{2}$ to higher temperatures than $400{ }^{\circ} \mathrm{C}$, yielding partial carbonization of the networks. ${ }^{41}$

Although CTFs are remarkable materials, it has to be mentioned that their synthesis is somewhat tedious. Due to the 
high temperatures applied, the reaction has to be conducted in evacuated and sealed quartz ampoules. In this respect care has to be taken that the organic monomers are not decomposing or that the (hygroscopic) $\mathrm{ZnCl}_{2}$ is applied in a dry state, as any evolving gases increase the pressure in the ampoule. It is therefore recommended to fill the ampoules with salt and monomer in a glove box - and to have a very diligent glass blower available for sealing them - before efficient synthesis and research on CTFs can be conducted. To circumvent these problems CTF materials were also prepared in trifluoromethanesulfonic acid and microwave heating. ${ }^{42}$ However, neither the microwave nor the quartz ampoule approach can be easily applied for an upscaling of CTF synthesis. Herein we show that CTF synthesis in molten zinc chloride can be carried out in open ceramic crucibles when instead of the monomeric cyano-compound a pre-formed triazine polymer network is used, synthesized by trimerization of the cyano-compound in strong Broensted acids. Thus a two-step approach is introduced in which the first trimerization reaction is carried out at lower reaction temperatures and just the final polymerization and crystallization of the CTF occurs in the molten salt. This process is therefore much more convenient as it can be carried out without the use of glove boxes and sealed vessels. Such a facile synthesis might help to further stimulate the investigation of novel CTF structures or applications.

\section{Experimental section}

\section{Chemicals}

1,4-Dicyanobenzene (terephtalonitrile) was purchased from Fluka and used as received. Trifluoromethanesulfonic acid (TCI, >98\%), chlorosulfonic acid (Fluka, >98\%) and zinc chloride (Aldrich, Redi-Dry, anhydrous, 98\%, stored in a glove box) were used as received.

\section{General}

Thermogravimetric analyses (TGA) were carried out on a TGA 1 instrument from Mettler Toledo at $10 \mathrm{~K} \mathrm{~min}^{-1}$ under $\mathrm{N}_{2}$ or $\mathrm{O}_{2}$ atmosphere. IR spectra were collected on a Varian 640IR spectrometer equipped with an ATR cell. Powder X-ray diffractograms were performed in reflection mode on a Bruker D8 Advanced instrument with $\mathrm{Cu}-\mathrm{K}_{\alpha}$ radiation $(\lambda=0.154 \mathrm{~nm})$. Elemental analyses were obtained from a Thermo Flash EA 1112 Organic Elemental Analyzer. Solid-state NMR (cross-polarization magic-angle spinning (CP/MAS)) spectra were carried out on a Bruker Avance $400 \mathrm{MHz}$ spectrometer operating at $100.6 \mathrm{MHz}$ for ${ }^{13} \mathrm{C}$. Nitrogen sorption measurements were performed at $77 \mathrm{~K}$ using an Autosorb $\mathrm{IQ}_{2}$ from Quantachrome. The samples were degassed at $150{ }^{\circ} \mathrm{C}$ for $12 \mathrm{~h}$ before analysis. BET surface areas were determined over a range of $0.005-0.1 P / P_{0}$. Pore size distributions were calculated from nitrogen adsorption isotherms using a NLDFT model based on carbon/slit-cylindrical pores from ASiQwin software. A Nabertherm N7/H argon oven was used to heat the samples under inert atmosphere to the desired temperature.

\section{Synthesis of the acid catalyzed triazine network (pre-CTF)}

In an inert argon atmosphere $10 \mathrm{ml}$ of $\mathrm{CHCl}_{3}$ and trifluoromethanesulfonic acid $(6.00 \mathrm{~g}, 40.0 \mathrm{mmol})$ were added into a dry 2-neck round bottom flask. After cooling the mixture to $0{ }^{\circ} \mathrm{C}, 1,4$-dicyanobenzene $(1.28 \mathrm{~g}, 10.0 \mathrm{mmol})$ dissolved in $80 \mathrm{ml}$ $\mathrm{CHCl}_{3}$ was added slowly over 30 min under stirring. The reaction mixture was stirred for $2 \mathrm{~h}$ at $0{ }^{\circ} \mathrm{C}$ before heating it up to $40{ }^{\circ} \mathrm{C}$ and further stirring for $12 \mathrm{~h}$. The reaction mixture turned from colorless to yellow and a solid precipitate was formed. After cooling to RT, $200 \mathrm{ml}$ of deionized water containing $10 \mathrm{ml}$ of ammonia solution (25\%) was added and the mixture was stirred for $2 \mathrm{~h}$. The precipitate was isolated by vacuum filtration and washed successively with deionized water, EtOH, acetone and chloroform. The product was dried under vacuum for $12 \mathrm{~h}$ at $115{ }^{\circ} \mathrm{C}$. Pre-CTF $(0.55 \mathrm{~g}, 43 \%$ yield) was obtained as a yellow solid. FT-IR (ATR, $\mathrm{cm}^{-1}$ ): 2232, 1506, 1354, 811. Anal. calcd for $\mathrm{C}_{8} \mathrm{H}_{4} \mathrm{~N}_{2}$ : C, 74.99; N, 21.86; H, 3.15. Found: C, 68.67; N, 19.89; H, 3.13. See ESI $\mathrm{S} 1 \uparrow$ for detailed alternative trimerization protocols.

\section{Typical synthesis of CTF-1 material in open crucibles (CTF- 1_open_40 h)}

Pre-CTF ( $0.25 \mathrm{~g}$, corresponding to $1.95 \mathrm{mmol}$ reacted DCB units) was thoroughly mixed with $\mathrm{ZnCl}_{2}(0.22 \mathrm{~g}, 1.60 \mathrm{mmol})$ in an inert argon atmosphere. The mixture was transferred to a porcelain crucible with lid and stored in a closed storage box to maintain the inert argon atmosphere. After preheating the argon oven for $30 \mathrm{~min}$ under a regulated argon flow of $4 \mathrm{l} \mathrm{min}^{-1}$ to $400{ }^{\circ} \mathrm{C}$, the crucible was quickly placed into the argon oven and heated within $5 \mathrm{~min}$ to $400{ }^{\circ} \mathrm{C}$ for $40 \mathrm{~h}$. The crude product was ground and stirred in deionized water $(150 \mathrm{ml})$ for $12 \mathrm{~h}$ at $60^{\circ} \mathrm{C}$, filtered and washed thoroughly with water to remove the majority of the salt. The mixture was further stirred in $0.1 \mathrm{M} \mathrm{HCl}(150 \mathrm{ml})$ for $12 \mathrm{~h}$ at $60{ }^{\circ} \mathrm{C}$ to remove the residual $\mathrm{ZnCl}_{2}$, filtered and subsequently washed with water and THF. The product was dried under vacuum for $12 \mathrm{~h}$ at $150{ }^{\circ} \mathrm{C}$. CTF-1_open_40 $\mathrm{h}(0.13 \mathrm{~g}, 52 \%$ yield) was obtained as a black solid. FT-IR (ATR, $\left.\mathrm{cm}^{-1}\right): 2232$, 1506, 1343, 807. Anal. calcd for $\mathrm{C}_{8} \mathrm{H}_{4} \mathrm{~N}_{2}$ : C, 74.99; N, 21.86; H, 3.15. Found: C, 71.32; N, 17.53; H, 2.84. See ESI $\dagger$ for detailed experimental and analytical details of all CTF-1_open materials.

\section{Results and discussion}

The main reason why CTF synthesis should be conducted in sealed reaction vessels is simply that most cyanocompounds, as for example 1,4-dicyanobenzene (DCB) used to prepare CTF-1, have low boiling or sublimation points and therefore evaporate before the zinc chloride is even melting (Fig. 1). Indeed when DCB is mixed with zinc chloride and heated to the reported reaction temperature $\left(400{ }^{\circ} \mathrm{C}\right)$ in an open crucible just a very tiny amount of a black, undefined residue is observed after salt removal. However, it can be reasoned that already the first oligomers of DCB prepared by trimerization should have a much higher sublimation/evaporation temperature and can therefore be possibly heated to higher temperatures without mass loss. We therefore investigated in a first step different trimerization protocols and the thermal stability of the 


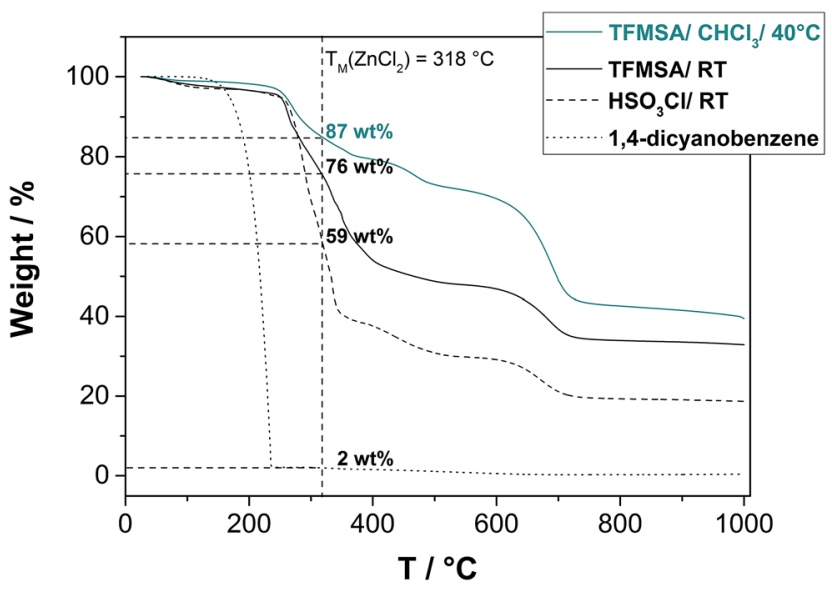

Fig. 1 TGA measurement of three different pre-CTFs and DCB. Shown is the observed weight loss at the melting temperature of $\mathrm{ZnCl}_{2}$ $\left(318^{\circ} \mathrm{C}\right)$.

resulting oligomers and triazine networks ("pre-CTFs"). For example when DCB was stirred in trifluoromethanesulfonic acid (TFMSA)/chloroform mixtures a light yellow precipitate appeared after several minutes, which was isolated and purified by filtering and washing. Fig. 1 shows the TGA curves from three different pre-CTFs prepared under different conditions and compared to the TGA curve of DCB. We especially focused on finding a pre-CTF with maximum stability at the melting temperature of $\mathrm{ZnCl}_{2}\left(318^{\circ} \mathrm{C}\right)$.

It was found that a pre-CTF prepared in chloroform/TFMSA at $40{ }^{\circ} \mathrm{C}$ showed the highest thermal stability and at $318{ }^{\circ} \mathrm{C}$ just a weight loss of $13 \mathrm{wt} \%$ is observed. Other acids, solvents and temperatures were tested (see experimental part and ESI $\dagger$ ) but no other combination yielded materials with higher thermal stability. Therefore this pre-CTF was chosen for further studies of structure development in molten $\mathrm{ZnCl}_{2}$. It should be noted that the pre-CTF can be easily made in large scales and the simple isolation and purification steps would even allow for recycling of the applied acid and solvent.

The pre-CTF was mixed with solid $\mathrm{ZnCl}_{2}$ and added to a ceramic crucible which was just covered (but not sealed) with a ceramic lid. For CTF-1 it was reported that highest crystallinity is observed using a monomer to $\mathrm{ZnCl}_{2}$ molar ratio of $1: 1$. Here a slight excess of the pre-CTF was chosen yielding a molar ratio of $1: 0.8$ of DCB units in pre-CTF to $\mathrm{ZnCl}_{2}$, considering the weight loss of the pre-CTF until $400{ }^{\circ} \mathrm{C}$ as observed by TGA (Fig. 1). The so-prepared pre-CTF/ $\mathrm{ZnCl}_{2}$ mixtures were in the first attempt heated to $400{ }^{\circ} \mathrm{C}$ with changing reaction times. After cooling down the mixtures and removal of $\mathrm{ZnCl}_{2}$ by water/ HCl washing, dark brown to black residues were obtained in approx. 50\% yields (Tables S1 and S2 $\dagger$ ). This yield is unsurprisingly smaller than observed for the approach in sealed ampoules, which is close to $100 \%$. However, this might be acceptable when relatively cheap monomers, like DCB, are employed, considering the much more convenient preparation protocol. It is furthermore notable, that the yields stay nearly constant for all reaction times, i.e. the major mass loss occurs already after short reaction times, probably by fast removal of thermally less stable side groups in the pre-CTF (see below).

The resulting CTF materials after 5-40 h reaction times were analyzed by means of XRD, BET, elemental analysis and ${ }^{13} \mathrm{C}-\mathrm{NMR}$ measurements and compared to pre-CTF and CTF-1 prepared in a sealed ampoule.

XRD measurements of the samples are shown in Fig. 2a. CTF-1 prepared in closed ampoules features a two-dimensional, layered structure, with hexagonal oriented pores reflected in the intense peak at lower angles $\left(7.5^{\circ}\right)$, attributed to the in-plane (100) reflection, while the broad peak at $\sim 25.5^{\circ}$ can be attributed to the interlayer (001) stacking. In contrast, pre-CTF shows no crystallinity. However, CTF-1 prepared from pre-CTF in open crucibles show comparable XRD patterns as observed for CTF-1 prepared in the sealed ampoule already after 5 hours reaction time. It can be thus reasoned that the application of the polymeric pre-CTF facilitates the formation of ordered CTF-1 compared to the use of dicyanobenzene monomers. This might be due to a preorganization of monomers within the pre-CTF, which can be seen in a less intense, very broad but already visible peak at low angles in the XRD of the pre-CTF. The order within the pre-CTF is possibly largely disturbed by defects, which number is however fast decreased after heating to $400{ }^{\circ} \mathrm{C}$. Over time the low angle (100) peak is increased in intensity compared to the interlayer (001) peak, which could point to an enhanced intralayer structuring with ongoing reaction time. This is supported by the corresponding nitrogen sorption measurements (Fig. 2b). While pre-CTF shows no detectable surface area, the increasing structural order of CTF-1 after different reaction times is reflected in an increasing surface area from $545 \mathrm{~m}^{2} \mathrm{~g}^{-1}(5 \mathrm{~h})$ to $910 \mathrm{~m}^{2} \mathrm{~g}^{-1}(40 \mathrm{~h})$ (Table S3†). This shows that after $40 \mathrm{~h}$ reaction time a CTF- 1 is observed with structural ordering and porosity well comparable to CTF-1 prepared in sealed quartz ampoules $\left(791 \mathrm{~m}^{2} \mathrm{~g}^{-1}\right)$. All nitrogen sorption isotherms show type I characteristics, typical for purely microporous materials. The observation that the desorption
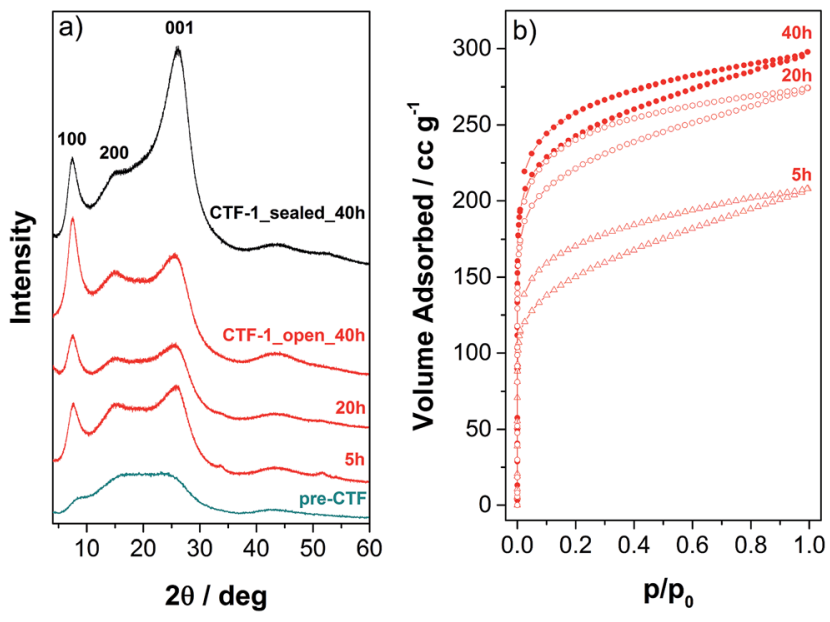

Fig. 2 (a) Powder XRD patterns and (b) nitrogen sorption measurements of pre-CTF, CTF-1 prepared in a sealed quartz ampoule and CTF-1 prepared from pre-CTF in open ceramic crucibles after different reaction times. 
branch is not closing on the adsorption branch and apparently showing a hysteresis has been frequently described for microporous polymer networks and is assigned to a partial swelling of the rather soft polymer networks under increasing nitrogen pressure. The calculated pore size distributions (Fig. S7 and S8†) show that all CTF materials are microporous, while two types of pores seem to be present with diameters of $1.2 \mathrm{~nm}$ and $0.6 \mathrm{~nm}$, respectively. While the former corresponds to the expected pore diameter for an ideal CTF-1 stacked in an eclipsed fashion, ${ }^{3}$ the smaller pores could be a result of a layer offset and stacking disorder, as such pore diameters have been calculated for the isoelectronic COF-1 structure when stacked in a staggered fashion. ${ }^{43}$

${ }^{13}$ C-MAS-NMR measurements (Fig. 3 ) of the CTF-1 materials show four distinct peaks which can be assigned to the carbon atoms in the triazine rings (169 ppm) and the two distinguishable carbons in the bridging benzene rings (136 and $126 \mathrm{ppm}$ ). A fourth peak at $114 \mathrm{ppm}$ can be assigned to terminal, nonreacted nitrile groups and is therefore a good qualitative measure for the degree of polymerization over time. Indeed it is seen that the intensity of this peak decreases with ongoing reaction time and is nearly comparable to the one observed for CTF- 1 from the sealed ampoule after $40 \mathrm{~h}$ reaction time. On the first glance it is surprising that for the pre-CTF this carbonitrile peak has also a low intensity, as the opposite would be expected due to the assumed lower polymerization degree. However, for pre-CTF additional peaks can be recognized, most prominent at $175 \mathrm{ppm}$, which can be assigned to carbonyls for example in amide groups. It can be therefore reasoned that during pre-CTF synthesis some terminal carbonitrile groups are transferred to amide or carboxyl groups with residual water. These groups might be also the first to decompose with increasing temperature, which could explain the fast mass loss after short reaction times, while, after these groups are vanished, the yield of the reaction stays nearly constant.

Table 1 shows the elemental analysis of the CTF materials compared in this study. For all CTF-materials slightly lower amounts of $\mathrm{C}, \mathrm{N}$ and $\mathrm{H}$ are observed than expected, which however is frequently described for CTFs and also other
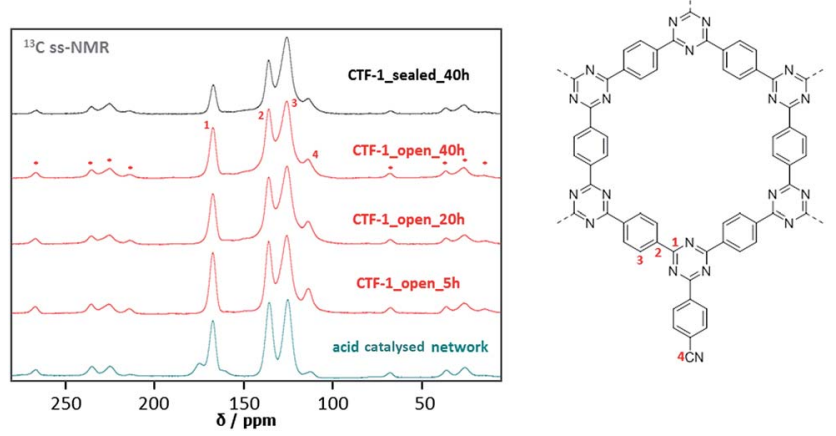

Fig. $3{ }^{13} \mathrm{C}$-MAS-NMR spectra of pre-CTF, CTF-1 prepared in a sealed quartz ampoule and CTF-1 prepared from pre-CTF in open ceramic crucibles after different reaction times and assignment on the observed peaks to the CTF structure.
Table 1 Elemental analysis of pre-CTF, and CTF-1 prepared in a sealed ampoule and in open crucibles after $40 \mathrm{~h}$

\begin{tabular}{lllll}
\hline & $\mathrm{C}[\mathrm{wt} \%]$ & $\mathrm{N}[\mathrm{wt} \%]$ & $\mathrm{C} / \mathrm{N}^{a}$ & $\mathrm{H}[\mathrm{wt} \%]$ \\
\hline${ }^{\mathrm{CTF}-1}$ (theor.) $_{\text {r }}$ & 75.00 & 21.86 & 4.00 & 3.15 \\
Pre-CTF & 68.67 & 19.89 & 4.03 & 3.13 \\
CTF-1 $_{\text {(sealed) }}$ & 71.83 & 19.54 & 4.29 & 3.02 \\
${ }_{\text {CTF-1 }}$ (open) & 71.32 & 17.53 & 4.75 & 2.84 \\
${ }^{a}$ Molar ratio. & & & & \\
\end{tabular}

microporous polymer networks and explained by incomplete burning of the materials, adsorbed water, residual catalysts or solvents trapped in the network. For pre-CTF also the formation of amide/carboxyl end groups as seen in the NMR measurement can explain the slightly increased hydrogen content.

The expected $\mathrm{C} / \mathrm{N}$ molar ratio of $4: 1$ is also nearly found in the pre-CTF but is slightly increased for CTF-1 prepared in the sealed ampoule and even more in the open crucible, showing that partial carbonization cannot be fully excluded by the salt melt approach. However, it can be concluded at this point that the two-step approach reported here, which enables the application of open crucibles for the salt melt reaction step, produces materials which are quite comparable to CTF-1 prepared in sealed ampoules. The here presented two-step approach also enables fast and parallel investigation and optimization of CTF-1 synthesis. As mentioned in the beginning, all CTF-1 materials reported so far were prepared using a molar ratio of $1: 0.8$ of pre-CTF to $\mathrm{ZnCl}_{2}$ based on the TGA measurement. However, to prove if this is indeed the optimum ratio, a series of pre-CTF $/ \mathrm{ZnCl}_{2}$ ratios was further tested. At this point the feasibility of our new synthesis approach is evidenced, as after preparing one large batch of pre-CTF, this can be used for a large number of reactions. For example, for this experiment the pre-CTF was parted and distributed to different crucibles with various amounts of $\mathrm{ZnCl}_{2}$ which were then heated in parallel to $400{ }^{\circ} \mathrm{C}$ for $40 \mathrm{~h}$.

The XRD measurements of the resulting CTF materials are shown in Fig. 4 and confirm the optimum molar ratio of preCTF to $\mathrm{ZnCl}_{2}$ of $1: 0.8$ to prepare an ordered CTF material, i.e. CTF-1 under the here chosen conditions. The intensity of the low angle peak (100) of the studied materials increases from 0.1 eq. $\mathrm{ZnCl}_{2}$ to 0.8 eq. $\mathrm{ZnCl}_{2}$, where the maximum intensity proves the highest intralayer order. Just a slight increase of the $\mathrm{ZnCl}_{2}$ content to 0.9 eq. leads to a loss of the hexagonal intralayer order, resulting in a drastically decreased intensity of the low angle peak. It has been frequently described that an equimolar salt to monomer ratio is crucial for the formation of ordered CTF materials, probably due to a templating effect of the $\mathrm{ZnCl}_{2}$. Furthermore, irreversible side reactions are promoted when an excess of $\mathrm{ZnCl}_{2}$ is used. This is also seen in the steady increase in surface area with increasing salt content, from $667 \mathrm{~m}^{2} \mathrm{~g}^{-1}$ (0.6 eq.) to $1377 \mathrm{~m}^{2} \mathrm{~g}^{-1}$ (1.5 eq.), which is in accordance to the synthesis carried out in sealed ampoules (see Fig. S4 and Table S3†). ${ }^{8,41}$ 


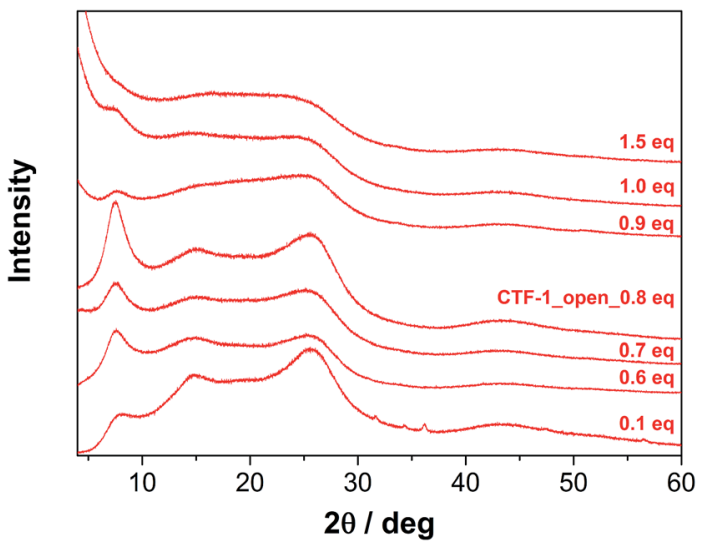

Fig. 4 Powder XRD patterns of CTF materials prepared from pre-CTF in open ceramic crucibles with different equivalents of $\mathrm{ZnCl}_{2}(0.1-1.5$ eq.) after $40 \mathrm{~h}$ reaction time.

For several applications it was reported that CTF materials heated to higher temperatures (e.g. $600{ }^{\circ} \mathrm{C}$, i.e. "TTFs") are suitable materials, as their surface area, pore size and conductivity increases in comparison to CTF-1, even though on expense of crystallinity as partial carbonization occurs, as seen in strongly increasing $\mathrm{C} / \mathrm{N}$ ratios. To test if the two-step approach is applicable for a large scope of CTF materials, a mixture of preCTF and $\mathrm{ZnCl}_{2}$ was this time heated up to $600{ }^{\circ} \mathrm{C}$, again in a non-sealed crucible. Indeed a material was observed with an increased surface area of $1551 \mathrm{~m}^{2} \mathrm{~g}^{-1}$ (Fig. S6 $\dagger$ ) and additional mesopores (Fig. S9†) which however shows no sign of crystallinity (Fig. S11†), thus again resembling the material prepared in closed quartz ampoules. ${ }^{41}$

While the transfer from evacuated and sealed quartz ampoules to open crucibles is a major simplification of the CTF synthesis, it should be noted that all presented materials were still mixed in a glove box and then transferred as fast as possible to the oven to avoid the presence of water during synthesis. To test if strict avoidance of moisture is indeed necessary and to probably further enhance the feasibility of the here shown approach, the $\mathrm{ZnCl}_{2}$ /pre-CTF mixture ( 0.8 eq.) was intentionally exposed to air moisture for $20 \mathrm{~min}$ (note that $\mathrm{ZnCl}_{2}$ is strongly hygroscopic thus that exposure to humid air should be strictly avoided when used in sealed quartz ampoules). The mixture was subsequently heated to $400{ }^{\circ} \mathrm{C}$ for $40 \mathrm{~h}$. After removal of $\mathrm{ZnCl}_{2}$ a material was obtained in just slightly lower yield than under "dry" conditions ( $50 \%$ vs. $52 \%$ ) and which showed also a comparable surface area and pore size distribution to CTF-1 (950 $\mathrm{m}^{2} \mathrm{~g}^{-1}$ vs. $910 \mathrm{~m}^{2} \mathrm{~g}^{-1}$ ) (Fig. S5, S10 and Table S3†). Furthermore XRD measurement revealed also for this material the characteristic pattern for CTF-1 (Fig. S12 $\dagger$ ), showing that the here presented synthesis can be even carried out with $\mathrm{ZnCl}_{2}$ which is not extensively dried before use.

\section{Conclusion}

Covalent triazine frameworks (CTFs) can be prepared in open crucibles, when instead of the cyanomonomers a pre-CTF, namely a triazine polymer network is applied. The pre-CTF can be easily prepared from the monomers in $\mathrm{CHCl}_{3}$ and triflouromethanesulfonic acid. When this amorphous and nonporous triazine polymer network is heated together with $\mathrm{ZnCl}_{2}$, a microporous, crystalline covalent triazine framework is obtained, which can be readily compared to the CTFs so far reported from the synthesis in sealed quartz ampoules. The here shown approach largely facilitates the synthesis of CTFs, which on the one hand should enable a faster and easier investigation and development of novel CTFs or CTF composites, but will be also of high importance for a possible upscaling of CTF material synthesis. It will be of interest if the here shown approach, i.e. the application of pre-formed polymeric precursors instead of small molecular compounds, can be also used for the synthesis of other known and novel covalent organic frameworks.

\section{Acknowledgements}

The authors acknowledge the support from the Sino-German Center for Research Promotion (GZ879) and the European Research Council (ERC) (Grant: 78593_ORGZEO).

\section{Notes and references}

1 A. Thomas, Angew. Chem., Int. Ed., 2010, 49, 8328-8344.

2 S.-Y. Ding and W. Wang, Chem. Soc. Rev., 2013, 42, 548-568.

3 P. Kuhn, M. Antonietti and A. Thomas, Angew. Chem., Int. Ed., 2008, 47, 3450-3453.

4 M. J. Bojdys, J. Jeromenok, A. Thomas and M. Antonietti, Adv. Mater., 2010, 22, 2202-2205.

5 P. Katekomol, J. Roeser, M. Bojdys, J. Weber and A. Thomas, Chem. Mater., 2013, 25, 1542-1548.

6 Y. Zhao, K. X. Yao, B. Teng, T. Zhang and Y. Han, Energy Environ. Sci., 2013, 6, 3684-3692.

7 G. Algara-Siller, N. Severin, S. Y. Chong, T. Bjorkman, R. G. Palgrave, A. Laybourn, M. Antonietti, Y. Z. Khimyak, A. V. Krasheninnikov, J. P. Rabe, U. Kaiser, A. I. Cooper, A. Thomas and M. J. Bojdys, Angew. Chem., Int. Ed., 2014, 53, 7450-7455.

8 P. Kuhn, A. Thomas and M. Antonietti, Macromolecules, 2009, 42, 319-326.

9 R. Palkovits, M. Antonietti, P. Kuhn, A. Thomas and F. Schuth, Angew. Chem., Int. Ed., 2009, 48, 6909-6912.

10 J. Artz, S. Mallmann and R. Palkovits, ChemSusChem, 2015, 8, 672-679.

11 A. V. Bavykina, M. G. Goesten, F. Kapteijn, M. Makkee and J. Gascon, ChemSusChem, 2015, 8, 809-812.

12 F. Chang, J. Guo, G. Wu, L. Liu, M. Zhang, T. He, P. Wang, P. Yu and P. Chen, RSC Adv., 2015, 5, 3605-3610.

13 C. E. Chan-Thaw, A. Villa, P. Katekomol, D. Su, A. Thomas and L. Prati, Nano Lett., 2010, 10, 537-541.

14 C. E. Chan-Thaw, A. Villa, L. Prati and A. Thomas, Chem.Eur. J., 2011, 17, 1052-1057.

15 C. E. Chan-Thaw, A. Villa, G. M. Veith, K. Kailasam, L. A. Adamczyk, R. R. Unocic, L. Prati and A. Thomas, Chem.-Asian J., 2012, 7, 387-393. 
16 S.-Y. Ding, J. Gao, Q. Wang, Y. Zhang, W.-G. Song, C.-Y. Su and W. Wang, J. Am. Chem. Soc., 2011, 133, 19816-19822.

17 R. Palkovits, C. von Malotki, M. Baumgarten, K. Muellen, C. Baltes, M. Antonietti, P. Kuhn, J. Weber, A. Thomas and F. Schueth, ChemSusChem, 2010, 3, 277-282.

18 M. Rose, ChemCatChem, 2014, 6, 1166-1182.

19 C. E. Chan-Thaw, A. Villa, D. Wang, V. Dal Santo, A. O. Biroli, G. M. Veith, A. Thomas and L. Prati, ChemCatChem, 2015, 7, 2149-2154.

20 K. Kamiya, R. Kamai, K. Hashimoto and S. Nakanishi, Nat. Commun., 2014, 5, 5040.

21 J. Roeser, K. Kailasam and A. Thomas, ChemSusChem, 2012, 5, 1793-1799.

22 L. Hao, S. Zhang, R. Liu, J. Ning, G. Zhang and L. Zhi, Adv. Mater., 2015, 27, 3190-3195.

23 J. Liu, Y. Hu and J. Cao, Catal. Commun., 2015, 66, 91-94.

24 A. Bhunia, I. Boldog, A. Moeller and C. Janiak, J. Mater. Chem. A, 2013, 1, 14990-14999.

25 A. Bhunia, V. Vasylyeva and C. Janiak, Chem. Commun., 2013, 49, 3961-3963.

26 X. Chen, F. Yuan, Q. Gu and X. Yu, J. Mater. Chem. A, 2013, 1, 11705-11710.

27 S. Hug, M. B. Mesch, H. Oh, N. Popp, M. Hirscher, J. Senker and B. V. Lotsch, J. Mater. Chem. A, 2014, 2, 5928-5936.

28 X. Liu, H. Li, Y. Zhang, B. Xu, A. Sigen, H. Xia and Y. Mu, Polym. Chem., 2013, 4, 2445-2448.

29 T. Wang, K. Kailasam, P. Xiao, G. Chen, L. Chen, L. Wang, J. Li and J. Zhu, Microporous Mesoporous Mater., 2014, 187, 63-70.

30 A. Bhunia, S. Dey, M. Bous, C. Zhang, W. von Rybinski and C. Janiak, Chem. Commun., 2015, 51, 484-486.
31 H. Ren, T. Ben, E. Wang, X. Jing, M. Xue, B. Liu, Y. Cui, S. Qiu and G. Zhu, Chem. Commun., 2010, 46, 291-293.

32 W. Suksaengrat and P. Srepusharawoot, Microelectron. Eng., 2013, 108, 192-194.

33 X. Jiang, P. Wang and J. Zhao, J. Mater. Chem. A, 2015, 3, 7750-7758.

34 F. Niu, L. Tao, Y. Deng, H. Gao, J. Liu and W. Song, New J. Chem., 2014, 38, 5695-5699.

35 L.-M. Tao, F. Niu, D. Zhang, T.-M. Wang and Q.-H. Wang, New J. Chem., 2014, 38, 2774-2777.

36 L. Hao, B. Luo, X. L. Li, M. H. Jin, Y. Fang, Z. H. Tang, Y. Y. Jia, M. H. Liang, A. Thomas, J. H. Yang and L. J. Zhi, Energy Environ. Sci., 2012, 5, 9747-9751.

37 L. Hao, J. Ning, B. Luo, B. Wang, Y. Zhang, Z. Tang, J. Yang, A. Thomas and L. Zhi, J. Am. Chem. Soc., 2015, 137, 219-225.

38 H. P. Liao, H. M. Ding, B. J. Li, X. P. Ai and C. Wang, J. Mater. Chem. A, 2014, 2, 8854-8858.

39 K. Sakaushi, E. Hosono, G. Nickerl, T. Gemming, H. Zhou, S. Kaskel and J. Eckert, Nat. Commun., 2013, 4, 1485.

40 K. Sakaushi, G. Nickerl, F. M. Wisser, D. Nishio-Hamane, E. Hosono, H. Zhou, S. Kaskel and J. Eckert, Angew. Chem., Int. Ed., 2012, 51, 7850-7854.

41 P. Kuhn, A. Forget, D. Su, A. Thomas and M. Antonietti, J. Am. Chem. Soc., 2008, 130, 13333-13337.

42 S. Ren, M. J. Bojdys, R. Dawson, A. Laybourn, Y. Z. Khimyak, D. J. Adams and A. I. Cooper, Adv. Mater., 2012, 24, 23572361.

43 J. L. Mendoza-Cortes, S. S. Han, H. Furukawa, O. M. Yaghi and W. A. Goddard III, J. Phys. Chem. A, 2010, 114, 1082410833. 